\title{
Comments on: Remarkable polyhedra related to set functions, games and capacities
}

\author{
Peter Sudhölter ${ }^{1}$
}

Published online: 27 May 2016

(C) Sociedad de Estadística e Investigación Operativa 2016

The general interest in studying polyhedra in a systematic way certainly goes back to Euclid, and modern results, like those collected in this survey, about polyhedra arising in special situations are still attractive.

A set function is a real function, the domain of which consists of sets. The author Michel Grabisch restricts his attention to domains that are nonempty subsets of the power set of a finite set $N$, i.e., an unrestricted set function on $N$ is a mapping $\xi: 2^{N} \rightarrow$ $\mathbb{R}$. Hence, as pointed out explicitly, set functions often appear in mathematics and optimization as pseudo-Boolean functions where a subset $S$ of $N$ is identified with its characteristic function $1_{S} \in\{0,1\}^{N}$. Moreover, $\xi$ is (the coalitional or characteristic function of) a transferable utility (TU) game used to model cooperative interactive multi-personal decision problems in the theory of cooperative games if $\xi(\emptyset)=0$, and it is a capacity, an important concept of decision theory, if, in addition, it is isotone (w.r.t. inclusion) and normalized (i.e., $\xi(N)=1$ ). Replacing $\{0,1\}^{N}$ in the domain of pseudo-Boolean functions by $\{0, \ldots, k\}^{N}$ yields $k$-choice games in the sense of Hsiao and Raghavan (1990) ${ }^{1}$ and $k$-ary capacities in the sense of Grabisch and Labreuche (2003).

This survey contains a lot of material concerning the polytopes of certain important subsets of ( $k$-ary) capacities and TU games as well as polyhedral sets assigned to a (restricted) game like the core and some extensions. I should like to mention only some of these results. The set of capacities (on $N$ ) is a polytope whose extreme points are

\footnotetext{
1 If not appearing below, then see References of the commented article.
}

This comment refers to the invited paper available at doi:10.1007/s11750-016-0421-4.

$凶$ Peter Sudhölter psu@sam.sdu.dk

1 Department of Business and Economics and COHERE, University of Southern Denmark, Campusvej 55, 5230 Odense M, Denmark 
the simple games. This result has a straightforward generalization to $k$-ary capacities. The famous characterization of a convex game in terms of the marginal vectors of its core (Shapley 1971 and Ichiishi 1981) is discussed in detail, and the author carefully explains that this result has appeared in several forms in the literature and, surprisingly for me as a game theorist, already in an article by Dempster (1967). Moreover, if $N$ is endowed with a partial order relation and if a game is restricted to those subsets that are downsets of this poset, then the core of this game with precedence constraints may no longer be a polytope like in the traditional case, but remains to be a polyhedral set that does not contain lines so that it may be represented by its extreme points and extreme rays. The author provides a full account of the state of the art for the core of games with precedence constraints.

This discussant fully agrees that the mentioned concepts of set functions, capacities, and TU games play a significant role in the areas of mathematics, operations research, and game theory. He has only one minor reservation, namely against the $k$-additive core (for $k \neq 1$ ) because, despite the explanations provided in Section 8.1, he does not see the game theoretical interpretation. However, the corresponding results are mathematically interesting.

Thus, the survey presents an impressively large variety of interesting results on polyhedral sets that have been discussed in the literature on set functions, in particular games and capacities, and on some extensions. Having been involved in the investigations to many of these results, the author has also very carefully investigated what were the fundamental contributions to this topic in the literature. He also includes, as precisely as necessary and as concisely as possible, the necessary definitions of some properties and representation possibilities of games, capacities, and extensions.

Such an article can nevertheless not provide a complete account of the aforementioned topics-e.g., the close relation between equation (2) and Owen (1972) multilinear extension of a game might be of interest in this context as well as some results of Derks and Kuipers (2002). However, the article is very useful for mathematicians, game theorists, computer scientists, theoretical economists, and other researchers for obtaining relevant knowledge and references about or for recalling the aforementioned important results and topics.

\section{References}

Derks JJM, Kuipers J (2002) On the number of extreme points of the core of a transferable utility game. In: Chapters in game theory, Vol. 31 of Theory Decis. Lib. Ser. C. Kluwer Academic Publishers, Boston, pp. 83-97

Owen G (1972) Multilinear extensions of games. Manag Sci 18:64-79 